\title{
Scanning gate microscopy of magnetic focusing in graphene devices: quantum vs. classical simulation
}

\author{
M. D. Petrović, S. P. Milovanović, and F. M. Peeters \\ Department of Physics, University of Antwerp, \\ Groenenborgerlaan 171, B-2020 Antwerp, Belgium
}

\begin{abstract}
We compare classical versus quantum electron transport in recently investigated magnetic focusing devices [S. Bhandari et al., Nano Lett. 16, 1690 (2016)] exposed to the perturbing potential of a scanning gate microscope (SGM). Using the Landauer-Büttiker formalism for a multi-terminal device, we calculate resistance maps that are obtained as the SGM tip is scanned over the sample. There are three unique regimes in which the scanning tip can operate (focusing, repelling, and mixed regime) which are investigated. Tip interacts mostly with electrons with cyclotron trajectories passing directly underneath it, leaving a trail of modified current density behind it. Other (indirect) trajectories become relevant when the tip is placed near the edges of the sample, and current is scattered between the tip and the edge. We also discuss possible explanations for spatial asymmetry of experimentally measured resistance maps, and connect it with specific configurations of the measuring probes.
\end{abstract}

\section{INTRODUCTION}

Up to now, scanning gate microscopy (SGM) has been successfully used to image local electron transport in various mesoscopic systems. In early applications, SGM probed the interference effects produced by microscopic disorder in graphene, $\stackrel{1,2}{1}$ and it was later applied to image electron-hole puddles originating from extrinsic local doping. $\frac{3}{-}$ In the later experiment,,$\frac{3}{=}$ the tip was coated with a dielectric and placed directly in contact with graphene. The advantage of this approach is that AFM topography scans could be performed simultaneously with SGM scans, and the tip could be additionally used to clean the sample. Scanning technique was used in Ref. 4 to study the spatial inhomogeneity of the local neutrality point, and to measure the efficiency of intentionally embedding (writing) charges in graphene. $\frac{5}{-}$ Tip-dependent resistance map of a narrow quantum point contact (QPC), presented in Ref. 6, revealed a significant resistance increase when the tip was placed directly above the sample. The technique is also suitable for the investigation of localization effects. For example, concentric conductance halos were observed in SGM maps scanned around localized states in graphene quantum dots,,, 8 narrow constrictions $, 9,10$ and enhanced conductance was reported in narrow nanoribbons. ${ }^{11}$ Scanning gate experiments in quantum point contacts were simulated in Ref. 12 .

In this paper, we investigate the scanning gate experiments of the magnetic focusing devices reported in Refs. 13 16. Before, similar magnetic focusing measurements were performed on semiconductor two-dimensional electron gas (2DEG) in parallel with the scanning technique. ${ }^{17}$ We model our device using both quantum and classical transport theory. Previously, classical simulation of such a focusing device was done in Ref. 13, and focusing without SGM tip was studied in Ref. 18. A recent paper by Kolasiński et al..$^{19}$ was the first to reproduce some of the experimental findings by applying a full quantum approach. Here, we implement the multi-terminal
Landauer-Büttiker formalism to calculate the non-local resistances. We additionally compare the resistance maps of 4-terminal and 6-terminal devices for different combinations of voltage probes. Due to the large size of the device, as compared to the electron wavelength, most of the simulated effects can already be captured by the computationally less demanding classical model. In respect to that, we confirm that the classical billiard model can be successfully used to describe transport of relativistic carriers in the presence of inhomogeneous potentials, if the linear graphene spectrum is properly implemented.

Our analysis of simulated SGM maps confirms and expands on some of the experimental observations reported in Refs. 13 16. We consider both positively and negatively charged tip, as well as a tip acting in the mixed regime. We show that the main resistance feature (e.g. the spatial area of reduced resistance) can be explained by considering geometric relations between the device boundaries, the tip position, and the circular cyclotron orbits. We also found that the finite width of the focusing leads can not be neglected. One of the novel results is that the SGM tip is mainly acting on a set of trajectories that directly connect the injector lead with the tip position. We show that the repelling tip leaves a shadow behind itself, which is mainly situated in areas delimited by two direct cyclotron orbits that connect the tip with the two corners of the injector lead. The specific shape of the tip shadow, which depends on the relative position between the tip and the injector lead, determines the shape of the low resistance region. Therefore, at the first focusing peak, the tip is imaging a specific subset of direct trajectories connecting the two focusing leads. This only applies for a repelling tip, or a tip operating in a mixed regime (repelling and focusing). For a tip acting as a focusing lens, the produced SGM maps do not show any significant change in the resistance, therefore they do not provide much useful information in characterization of transport. We additionally compare results between a 4-terminal and a 6-terminal device, and find them to be 
very similar, although the later induces some asymmetry in the resistance maps. The reason for this similarity is because the resistance is mostly determined by the transmission function between the two focusing leads. In that sense, we confirm the approach taken in Ref. 13, which considered only transmission functions and not resistances.

This paper is organized as follows. In Sec. II we describe the focusing system, and indicate how we model the tip potential. Next, in Sec. IV], we scale the graphene tight-binding Hamiltonian and compare relevant quantities, such as the dispersion relation and the current density in a scaled and an unscaled graphene lattice. The resistance of an unperturbed device (i.e a device without the SGM tip) is studied in Sec. $\nabla$, while the analysis of SGM scans is done in Sec. V] In Sec. VI we present results of a six-terminal device, and discuss the possible causes of spatial asymmetry seen in the experiments. A short summary of our findings is given in Sec. VII]

\section{FOCUSING SYSTEM AND SGM POTENTIAL}

The studied system is shown in Fig. 1 It is a fourterminal graphene device with the same dimensions as those used in Ref. 13. The only major difference between our system, and that of Ref. 13, is the absence of two upper leads. We implement these two leads in Sec. VI. and discuss the changes they introduce in the SGM maps.

Magnetic focusing occurs when electron trajectories from the $2^{\text {nd }}$ lead (red curves in Fig. 1) are bent due to an external magnetic field into the $3^{\text {rd }}$ lead. Depending on the ratio between the width of the $3^{\text {rd }}$ lead $\left(l_{R}\right)$ and the distance between the leads $(L)$, the diverted electron can exit into the $3^{\text {rd }}$ or the $4^{\text {th }}$ lead. This switching of the exit lead manifests itself as oscillations in the device resistance. Figure 1 shows two focusing orbits, where an even number of cyclotron radii matches the separation between the leads, $2 n R_{c}=\left[L+\left(l_{R}+l_{L}\right) / 2\right]$. Focusing occurs as long as $2 R_{c}>l_{R}$.

Although magnetic focusing is a local phenomena, which depends on specific paths an electron can take in the system, it is usually studied by measuring the resistance of the whole device. A lot of information is lost in such measurements (e.g. the most probable electron trajectories). This spatial information can be retained by scanning-gate measurements, where a SGM tip perturbs the circular electron trajectories, causing the device conductance to become tip-dependent. The conductance maps produced in such measurements reveal how device conductance depends locally on electron passage through that point.

According to Refs. 13 and 14, a charged STM tip placed above a graphene sheet modifies the local charge

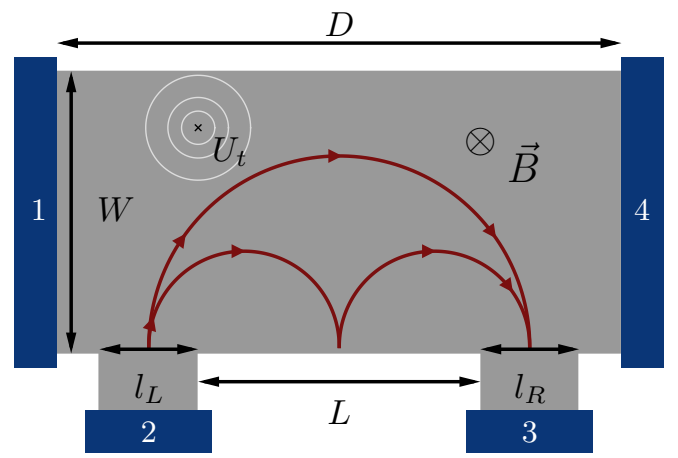

FIG. 1. Graphene magnetic focusing device: the system width is $W=2 \mu \mathrm{m}$, while the system length is $D=4 \mu \mathrm{m}$. Both horizontal (armchair), and vertical (zigzag) leads are metallic. The vertical leads have the same width $l_{L}=l_{R}=0.7 \mu \mathrm{m}$, and their separation is $L=2 \mu \mathrm{m}$. The magnetic field is perpendicular to the graphene sheet and points in the negative $z$ direction. The potential profile of the AFM tip is schematically represented by the white circles.

density in graphene

$$
\Delta n(\vec{r})=\frac{\tilde{q} h}{\left(d(\vec{r})^{2}+h^{2}\right)^{3 / 2}}
$$

which depends on the tip relative charge $\tilde{q}=-q / 2 \pi e$ (here $q$ is the actual charge accumulated on the tip, and $e$ is the electron charge), the distance from the tip to the graphene plane $h$, and the distance from the tip in-plane projection to the current point $\vec{d}(\vec{r})=\vec{r}-\vec{r}_{\text {tip }}$. A local change in the charge density modifies the local Fermi energy

$$
\Delta E_{F}(\vec{r})=E_{F}(n)-E_{F}(n+\Delta n(\vec{r})),
$$

which manifests as an additional tip-induced potential $U_{t}(\vec{r})=\Delta E_{F}(\vec{r})$. Since Fermi energy in graphene depends on the charge density through $E_{F}(n)=\hbar v_{F} \sqrt{\pi n}$, the induced potential is

$$
U_{t}(\vec{r})=\hbar v_{F} \sqrt{\pi}(\sqrt{n}-\sqrt{n+\Delta n(\vec{r})}) .
$$

This potential is a function of the global charge density $n$, and the local charge modification $\Delta n(\vec{r})$. The global density $n$ is set by the back-gate voltage, while the local modification $\Delta n(\vec{r})$ is determined by the tip height and the tip charge.

An alternative way to look into the perturbation of the tip is through the tip-generated force field. Charge carriers that travel through the system experience a force $\vec{F}(\vec{r})=-\vec{\nabla} U_{t}(\vec{r})$ generated by the tip. This force modifies the carrier equation of motion $\frac{13}{13}$

$$
\frac{d^{2} \vec{r}}{d t^{2}}=\frac{\vec{F}(\vec{r})}{m^{*}}=\frac{1}{2} v_{F}^{2} \frac{\nabla \tilde{n}(\vec{r})}{\tilde{n}(\vec{r})},
$$




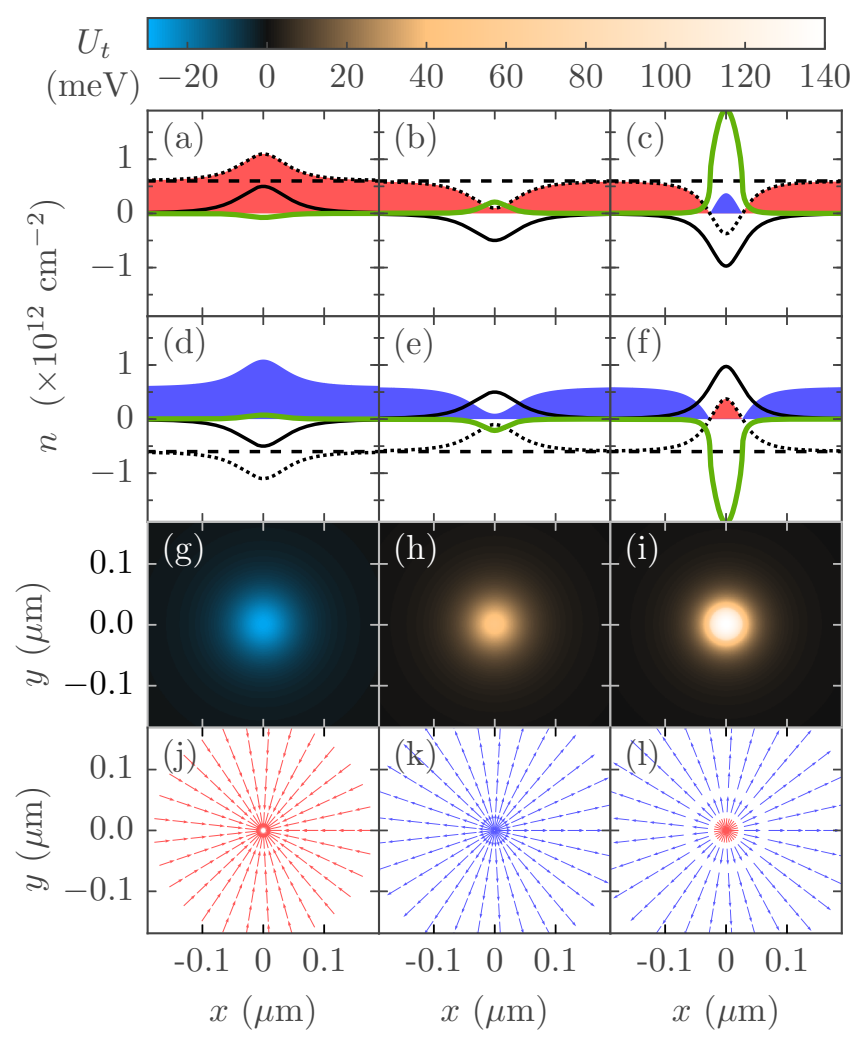

FIG. 2. Tip-induced potential: (a)-(f) Charge density beneath the tip $\left(x_{\text {tip }}=y_{\text {tip }}=0\right)$. Solid black curves show the local charge modification $\Delta n(\vec{r})$, while dashed black lines mark the global (unperturbed) charge density $n$. Dotted black curves show the resulting density $\tilde{n}(r)=n+\Delta n(r)$. Colored areas present the absolute density $|\tilde{n}(r)|$ for electrons (red) and holes (blue), while induced potential, expressed in charge density units $U_{t}^{2} /\left(\hbar^{2} v_{F}^{2} \pi\right)$, is shown by the green curves. (g)(i) Tip-induced potential for three regimes in (a), (b), and (c), respectively. (j)-(l) Directions of the force field acting on the charge carriers: inward (red), and outward (blue), for the corresponding potentials in $(\mathrm{g}),(\mathrm{h})$, and (i), respectively.

where $m^{*}$ is the carrier dynamical mass in graphene $\left(m^{*}=\hbar \sqrt{\pi n} / v_{F}\right)$, and $\tilde{n}(r)=n+\Delta n(r)$ is the resulting charge density.

Fig. 2] shows tip-induced potentials for different combinations of the global charge density and the local charge modification. There are six different regimes in which the tip can operate, but only three of these are unique. The other three regimes can be obtained by exchanging electrons with holes. The first regime is presented in Fig. 2(a). Here, a positively charged tip increases the local electron density, which manifests in the negative potential profile shown in Fig. 2(g). The tip-induced force field in Fig. 2(j) reveals a focusing nature of the tip. The case of negatively charged tip in Fig. 2(b) was previously studied experimentally. 13 As shown in Ref. 13 and in Fig. 2(h), the tip creates a positive potential which then repels the incoming electrons. The force field in this regime, shown in Fig. 2(k), is pointing away from

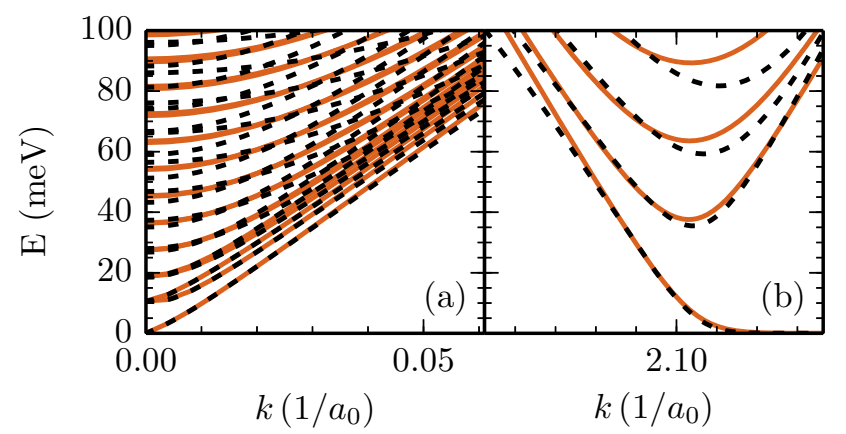

FIG. 3. Dispersion relations of the $1^{\text {st }}$ (a) and the $2^{\text {nd }}(b)$ lead. Magnetic field is $B=0.1 \mathrm{~T}$. Energy bands of pristine graphene are shown in orange, while those of the scaled graphene $\left(s_{f}=15.15\right)$ are shown by the black dashed curves. System dimensions are ten times smaller than those used in Fig. 1. Since the scaling procedure modifies the inverse $(k)$ space, we translated the K-point of the scaled system in (b), to match it with the K-point of the pristine graphene lattice.

the tip. In both Figs. 2(a) and 2(b), we set $|\Delta n(\vec{r})|$ to $5 \times 10^{11} \mathrm{~cm}^{-2}$. This density was used in Ref. 13 to fit the experimental data, and it corresponds to a tip positioned $h=60 \mathrm{~nm}$ above the graphene sheet. Assuming that the tip charge $\tilde{q}$ does not depend on the tip height $h$, in the far-left column of Fig. 2, we present results for a tip positioned closer to the sample $(h=43 \mathrm{~nm})$. When changing the tip height, we first calculate the tip charge $\tilde{q}$ from the modified charge density $\Delta n\left(r_{\text {tip }}\right)=\tilde{q} / h^{2}$, and then we recalculate $\Delta n$ for the new height.

Contrary to a classical 2DEG, where the tip depletes the electron density beneath it, $\stackrel{20}{=}$ in graphene, due to its gapless nature, the depleted electrons turn into holes. The induced potential in this third (or mixed) regime is much stronger than in both, the focusing and repelling regimes (compare Fig. 2(i) with Figs. 2(g) and2(h)). The force field is also specific (see Fig. 2(1)): in $n$ region (surrounding the tip) the tip repels incoming electrons, while in $p$ region (beneath the tip) the tip focuses tunneling holes. The actual tip height used in the experiment 13 could not go below $50 \mathrm{~nm}$ (because of the $50 \mathrm{~nm}$ thick BN layer separating the tip and the graphene sample). However, this does not mean that the third scanning regime is experimentally inaccessible. Enhanced tip potentials can be realized by lowering the global electron density $n$, or by increasing the tip charge $\tilde{q}$.

As stated above, we model this system from two perspectives, the quantum and the classical one. The classical billiard model is the same as that used in Ref. 18, while the quantum simulations are performed using KWANT, 21 a software package for quantum transport. Magnetic field in the quantum model is implemented through Peierls phase, as explained in Ref. 22, and the resistances are obtained by applying the Landauer-Büttiker formalism for a four-terminal ${ }^{23}$ and multi-terminal ${ }^{24}$ device. 


\section{SCALING THE TIGHT-BINDING HAMILTONIAN}

In order to simulate devices of similar sizes as those used in the experiment, 13 we scale the graphene tightbinding Hamiltonian. As explained in Ref. 25, a scaling coefficient $s_{f}$ is introduced. This coefficient increases the spacing between carbon atoms $a=s_{f} a_{0}$, and simultaneously decreases the nearest neighbor hopping energy $t=t_{0} / s_{f}$. The scaling procedure allows for simulations of systems with dimensions comparable to those used in actual experiments (in order of microns), but with lesser number of tight-binding orbitals. However, the scaling has its limits. Results for larger $s_{f}$ are less accurate, particularly for higher energies, away from the linear part of the spectrum. Close to the Dirac point, the scaled system is still a good approximation of the pristine graphene lattice.

To test the validity of the scaling procedure, we compare in Fig. 3 the dispersion relations obtained using scaled Hamiltonian with those obtained using the pristine graphene lattice. Since performing tight-binding calculations on a micrometer scale is computationally very demanding, we test the scaling procedure on a system ten times smaller than that presented in Fig. 11 The lattice scale of this smaller system $\left(s_{f}=15.15\right)$ is comparable to the lattice scale we use in the rest of the paper to simulate the micrometer-sized system shown in Fig. 1 ( $\left(s_{f}=15.34\right)$. As expected, the scaled dispersions match the pristine graphene lattice dispersions for low energies (below $100 \mathrm{meV}$ ). Although the band minimal energies differ, the scaled lattice is a good approximation for states away from the subband minima. Note that the scale is chosen such that it preserves the metallic nature of the armchair leads, as is seen in Fig. 3(a).

Since SGM experiments probe the local properties, it is necessary to determine how scaling affects them. With that in mind, in Fig. 4 we compare two current densities: one for scaled, and one for unscaled lattice. Beside the loss in resolution, caused by a lesser number of carbon atoms, the general current flow patterns are preserved with scaling, which confirms that this method can be used to simulate SGM experiments.

\section{MAGNETIC FOCUSING}

We investigate now the general transport properties of the focusing device when no SGM tip is present. We are interested in how the focusing resistance $\left(R_{m}=\right.$ $\left.R_{12,43}\right)$ changes as a function of the magnetic field $B$, and the electron density $n$ (or Fermi energy). Ref. 13 reported several resistance peaks as a function of the applied field, related with the current focusing (see Fig. 2(a) in Ref. 13). We simulate these measurements, and in Fig. [5]we present a comparison of the resistances obtained from both, quantum and classical simulation. To match our colormaps with those of Ref. 13, in both cases, we

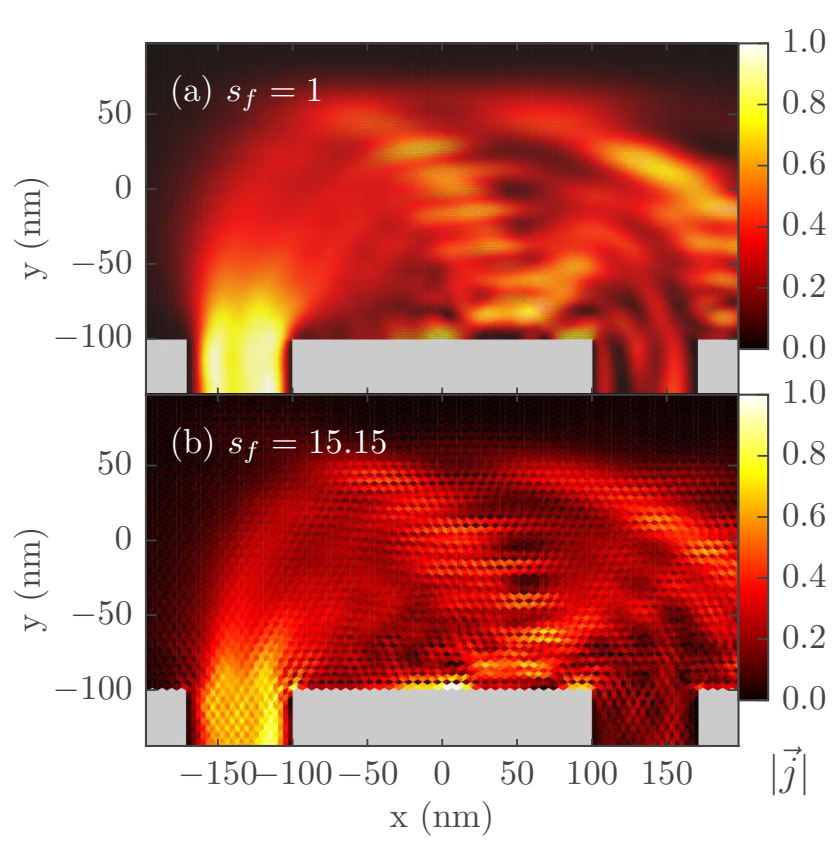

FIG. 4. (Color online) Normalized current density when current is injected from the second lead: (a) pristine graphene lattice $\left(s_{f}=1\right)$, and (b) scaled graphene lattice $\left(s_{f}=15.15\right)$. Magnetic field is $B=1 \mathrm{~T}$, and Fermi energy is $E_{F}=80 \mathrm{meV}$. The presented system is ten times smaller than that in Fig. 1] SGM tip is not present.

subtract $R_{0}=28 \Omega$ from numerically calculated $R_{12,43}$. This value is very close to $\left(R_{m}^{\max }+R_{m}^{\min }\right) / 2$. The resistances obtained with the two methods agree both qualitatively and quantitatively. The only difference is that resistances obtained with the quantum method show a set of parabolic fringe lines at higher fields, coming from Landau quantization. The classical method does not account for transversal quantization in the leads, and for the existence of transverse modes, hence the transmissions obtained classically need to be properly scaled before resistance calculations. We perform this scaling by multiplying the classically obtained transmissions with the approximate number of modes in the source lead 26

$$
M_{i}=2 \frac{E_{F} W_{i}}{\hbar v_{F} \pi} .
$$

Here index $i$ refers to the leads, $W_{i}$ is the width of the $i$-th lead, $E_{F}$ is the Fermi energy in the $i$-th lead, $v_{F}$ is the graphene Fermi velocity, while coefficient 2 is added to account for contributions coming from the two valleys. The graphene Fermi velocity $v_{F}$ in the tight-binding model is determined (to first approximation) from the nearest-neighbour hopping energy as $v_{F}=3|t| a / 2 \hbar$, where $a$ is the distance between neighbouring carbon atoms. For $t=2.7 \mathrm{eV}$, and $a=1.42 \AA$ we obtain $v_{F}=873893 \mathrm{~m} / \mathrm{s}$, which is the value that we use in our classical model.

Since cyclotron radius in graphene is proportional to the Fermi energy $R_{c}=E /\left(e v_{F} B\right)$, and Fermi energy 


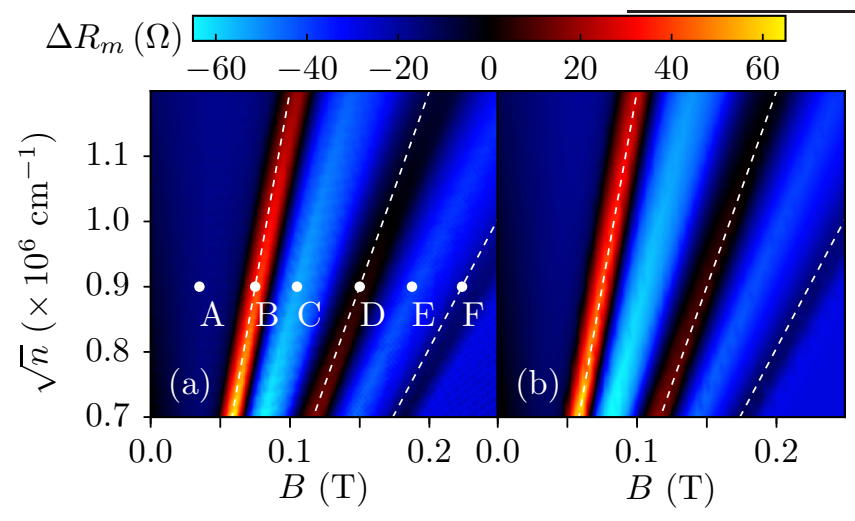

FIG. 5. (Color online) Change of the $R_{m}=R_{12,43}$ resistance versus the magnetic field and the electron density (Fermi energy) obtained from (a) quantum, and (b) classical simulation. In order to be able to compare both results with those published in Ref. 13, we calculate $\Delta R_{m}$ by subtracting $R_{0}=28 \Omega$ from numerically obtained $R_{12,43}$. The white dashed lines mark the first three focusing maxima, and they correspond to cyclotron radii of $1.4 \mu \mathrm{m}, 0.7 \mu \mathrm{m}$, and $0.47 \mu \mathrm{m}$. Labels A$\mathrm{F}$ mark the $(\sqrt{n}, B)$ points for which we present the current density in Fig. 6

is proportional to carrier density $E=\hbar v_{F} \sqrt{\pi n}$, then $\sqrt{n}=\gamma R_{c} B$ (where $\gamma=e /(\hbar \sqrt{\pi})$ ). In other words, for equiradial lines, $\sqrt{n}$ is a linear function of the applied magnetic field. In Fig. 5 we mark three such lines (white dashed lines) for three cyclotron radii $(1.4 \mu \mathrm{m}, 0.7 \mu \mathrm{m}$, and $0.47 \mu \mathrm{m}$ ) in order to match the first three focusing maxima.

For narrow focusing leads (leads 2 and 3), resistance peaks appear each time a multiple of a cyclotron diameter $2 R_{c}$ matches the lead distance $L$. However, if the lead widths $\left(l_{R}\right.$ and $\left.l_{L}\right)$ are comparable to the distance between them, it is reasonable to assume that focusing would occur only when electron injected from the middle of the input lead exits in the middle of the output lead. Two such trajectories are presented in Fig. 11. A simplified focusing formula, which includes the lead widths, would then be $2 n R_{c}=(L+l)$. For $L=2 \mu \mathrm{m}$ and $l=0.7 \mu \mathrm{m}$, the first three focusing radii are $1.35 \mu \mathrm{m}$, $0.675 \mu \mathrm{m}$ and $0.45 \mu \mathrm{m}$, which approximately matches the three lines shown in Fig. 5. For cyclotron radii smaller than half of the lead width $(0.35 \mu \mathrm{m})$ focusing is no longer possible.

To test whether the three resistance peaks in Fig. 5 appear due to the current focusing, we show in Fig. 6 the local current density for electrons coming from the $2^{\text {nd }}$ lead, for points A-F marked in Fig. I(a). The current is obtained using the quantum (tight-binding) model. Insets B, D, and F indeed show high current concentration in the exiting $\left(3^{\text {rd }}\right)$ lead, which confirms our assumption. For stronger fields (insets D, E, and F), the current flows close to the system lower edge (the left edge in Fig. 6), in an area one cyclotron diameter wide, The reason why current spreads in an area one cyclotron diameter wide,

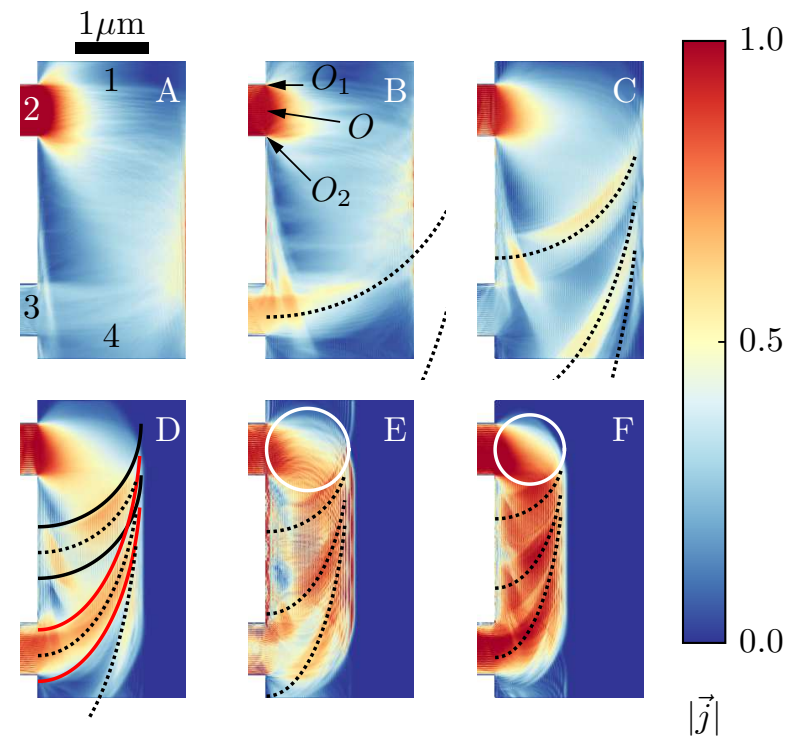

FIG. 6. (Color online) Normalized total current density at different focusing fields: (A) $35 \mathrm{mT}$, (B) $75 \mathrm{mT}$, (C) $105 \mathrm{mT}$, (D) $150 \mathrm{mT}$, (E) $188 \mathrm{mT}$, and (F) $223 \mathrm{mT}$ (points A-F marked in Fig. 5). The current is calculated using the quantum method. The electron density is $n=8.1 \times 10^{11} \mathrm{~cm}^{-2}$. Lead numbers for this rotated system are shown in figure A. Black-dashed curves show elliptical trajectories given by Eq. (6), and centered on the middle of the $2^{\text {nd }}$ lead (point $O$ ). Black and red full curves show the same trajectories, but now centered at the corners of the $2^{\text {nd }}$ lead (points $O_{1}$ and $O_{2}$ ). White circles in $\mathrm{E}$ and $\mathrm{F}$ show cyclotron orbits for these two figures.

and not one cyclotron radius wide, is the following. In the classical picture, electrons which enter the system perpendicularly to the lower edge (i.e. parallel to the focusing leads) would spread approximately one cyclotron radius away from the edge, since their trajectories consist of semicircles (see the two trajectories in Fig. 11). On the other hand, electrons entering the system almost parallel to the lower edge (i.e. normal to the focusing lead direction) would make almost a full circle before they scatter on the lower edge (see two white circles in insets $\mathrm{E}$ and $\mathrm{F}$ in Fig. (6). The current then spreads in a diameter-wide area due to these electrons. For weak fields, most of these electrons do not even make a full orbit, since they exit into the $1^{\text {st }}$ lead (see insets A and B in Fig. 6, where one part of the current from the $2^{\text {nd }}$ lead exits into the $1^{\text {st }}$ lead).

Current in the system can be understood in terms of the cyclotron orbits. Based on the picture of classical trajectories presented in Ref. 27, we plot three envelope curves (black-dotted lines) to mark three paths where (according to the classical picture) the current is supposed to travel. Each of these three curves is a part of an elliptical line

$$
\left(x-x_{0}\right)^{2}-\left(\frac{y-y_{0}}{2 n}\right)^{2}=R_{c}^{2}, \quad n=1,2,3, \ldots
$$




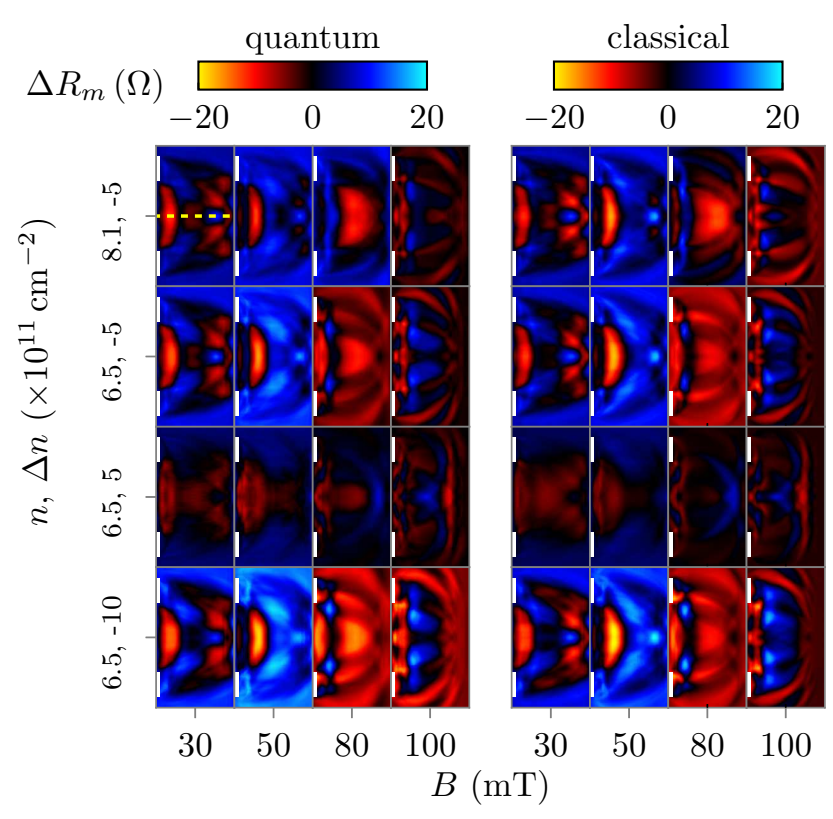

FIG. 7. (Color online) Comparison of the SGM resistance maps obtained from quantum (left) and classical (right) simulation. The two white stripes in the figures mark the positions of the $2^{\text {nd }}$ and the $3^{\text {rd }}$ lead. Labels on the left mark the electron density $n$, and maximal change in charge density introduced by the tip $\Delta n$. $\Delta R_{m}$ is calculated by subtracting $R_{0}=\left(R_{12,43}^{\max }+R_{12,43}^{\min }\right) / 2$ from $R_{12,43}$ for each scan. Yellowdashed line in the first row marks the mirror symmetry axis.

centered on the middle of the input lead $\left(x_{0}, y_{0}\right)=O$ (see point $O$ in Fig. 6B). It is clear that focusing occurs in insets $\mathrm{B}, \mathrm{D}$, and $\mathrm{F}$, since for these insets the elliptic curves pass through the $3^{\text {rd }}$ lead. The input lead has a finite width, hence these trajectories spread in space. The spread of current density is delimited by elliptical curves defined by Eq. (6) (black and red full curves in Fig. 6D), but now centered on two corners of the input lead (see points $O_{1}$ and $O_{2}$ in Fig. 6 $\mathrm{B}$ ).

\section{SCANNING GATE MICROSCOPY}

In this part we compare scanning gate maps obtained using the two models. We mentioned above that there are three regimes in which the tip can operate, therefore we additionally compare how scanning maps change in these three regimes. This comparison is presented in Fig. 7 In general, the local feature of the SGM maps obtained with the two models match. This is expected since the system size is larger than the electron wavelength, hence some of the interference effects are suppressed. The figure also confirms that on these scales a computationally less demanding classical model manages to capture all the features obtained with a more detailed atomistic model.

The first two rows in Fig. [7 show SGM maps for a repulsive (negatively charged) tip, as it was used in the experiment $\underline{13}$ The calculated resistances are very similar to the measured ones $\stackrel{13}{\underline{1}}$ and the main difference is that our results posses some extra oscillations close to the upper edge (shown on the right side in the rotated system in Fig. 7). As we show below, these features originate from multiple electron scatterings between the tip and the upper edge. The defining characteristic of all SGM maps obtained with repelling tip is a region of suppressed resistance close to the lower edge. This region evolves as magnetic field is increased and as shown in recent experiments, it can be connected with the cyclotron radius $\frac{15}{15}$

Resistance maps obtained with the focusing (positively charged) tip (third row in Fig. 7) convey less information than those obtained with a repelling tip. Although the tip causes some change in the resistance, some other effects, e.g temperature smearing, would even more degrade the obtained resistance maps. Therefore a tip in the focusing regime is probably not the best choice to probe electron transport. The third (mixed) regime seems to produce the largest change in the resistance: $R$ maps obtained in this regime show almost identical features as those obtained with a repelling tip, but the sample response is much better due to a stronger repelling force.

Previous (classical) simulations of magnetic focusing 13 considered only electron transmissions between the two focusing leads, and not the resistances. Here, we verify that this approach is valid. In Fig. 8 we compare the transmission maps between the two focusing leads $\left(T_{32}\right.$, left column) with the current densities (columns 2-4) for some specific tip positions (white and green circles). Calculated transmissions in the $1^{\text {st }}$ column of Fig. 8 resemble the corresponding $R$-maps in Fig. 7 (columns for $B=50$ $\mathrm{mT}$ in Fig. (7). Most of the features of the resistance maps are determined by the tip position relative to the two corners of the injector lead. In each of the insets 3 5 , and 8-10, we show two cyclotron orbits that directly connect the tip with the two corners (two white arcs in each of these insets). As shown in these insets, a trail of suppressed current is seen as a shadow that the tip leaves behind itself. The shadow is situated mostly in the area enclosed between the two cyclotron orbits. These two orbits (originating at two corners of the $2^{\text {nd }}$ lead) mark the boundary of a set of direct cyclotron trajectories that connect the tip with the $2^{\text {nd }}$ lead. The current is suppressed in these areas, because the tip blocks these trajectories. The diverted current forms an arc around the tip, and it flows away from the blocked area (see the two red arrows in inset 9, showing the flow direction of the diverted current). Similar explanation for the diverted current is given in Ref. 16. Our interpretation (based on the two delimiting orbits) also explains the areas of strongly suppressed resistance. Insets 7 and 8 show two positions of the tip lying on the edge of the resistance suppressed region (see inset 6 ). For any point lying on a line between these two points, the trail of blocked current coincides with the $3^{\text {rd }}$ lead. From these simple geometric relations, we see that the area of suppressed conductance 


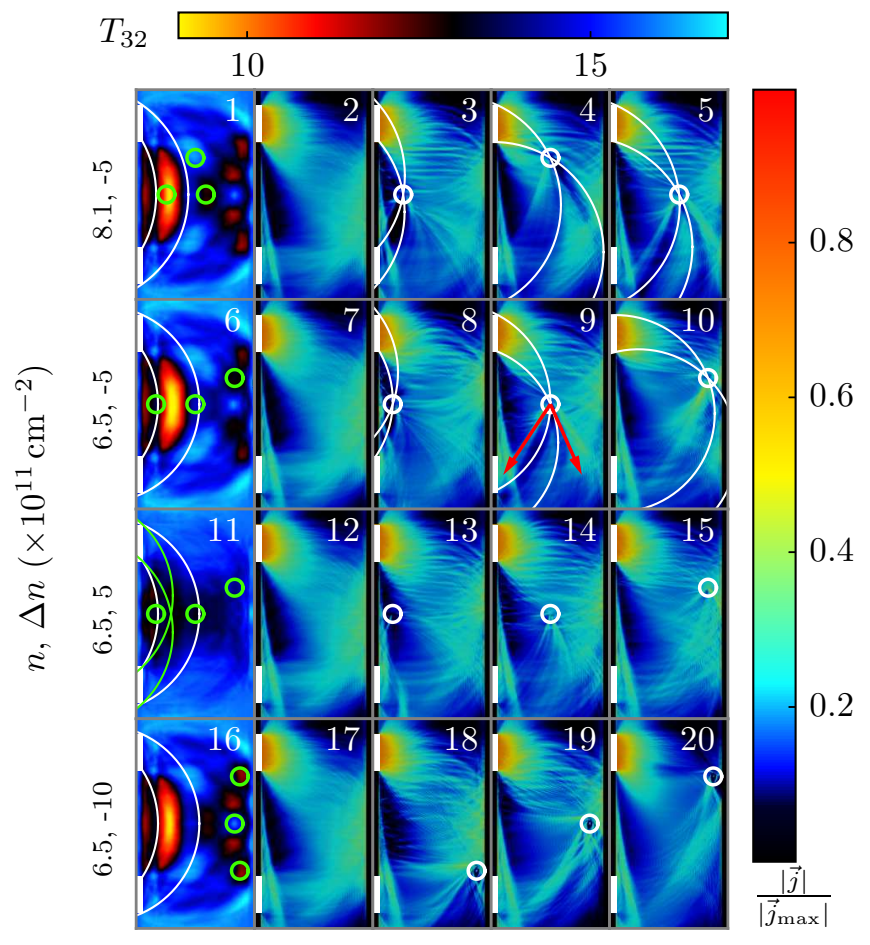

FIG. 8. (Color online) Comparison between electron transmission maps ( $1^{\text {st }}$ column on the left) and normalized electron current densities (columns 2-5 counted from the left). In the second column (insets $2,7,12,17$ ) we present current density with no SGM tip present, and in the rest (last three columns) we show current densities for specific tip positions (white circles). These tip positions are also marked in the first column (green circles) for comparison. The four rows correspond to the same four $n, \Delta n$ configurations as used in Fig. [7. Magnetic field is $B=50 \mathrm{mT}$. Again, the two focusing leads are marked in each inset with two white rectangles. In insets $3-5$, and $8-10$ we mark the direct trajectories connecting corners of the $2^{\text {nd }}$ lead with the tip position. The corresponding cyclotron radii for the two group of lines are $2.1 \mu \mathrm{m}$ (first row), and $1.88 \mu \mathrm{m}$ (second row). The white lines in the first column delimit the areas relevant for focusing.

is delimited by two cyclotron orbits that directly connect inner and outer corners of the focusing leads (white curves in the $1^{\text {st }}$ column of Fig. 8). The maximal resistance suppression is expected at the crossing point of orbits connecting inner and outer corners (see the two green curves in inset 11, and also the two cyclotron orbits in inset 3). Based on the previous analysis, we conclude that the finite width of the suppressed resistance region is an indirect consequence of the finite width of the two focusing leads.

The results for a focusing tip reveal effects opposite to those of the repelling tip (compare the tip influence on the current densities in the second and the third row in Fig. 8). The focusing tip leaves a trail of enhanced current instead of a shadow, as seen in the case of a repelling tip (compare insets 10 and 15). In the last row, a tip in the mixed regime shows a much darker shadow behind itself as compared to the repelling tip. The mixed nature of the tip manifests itself in a current profile, where some of the current that manages to tunnel through the potential induced by the tip, exits focused on the other side.

\section{RESISTANCE MAPS OF A SIX-TERMINAL DEVICE}

Although previous resistance maps capture the main features reported in the experiment $\underline{13}$ (e.g. they show semicircular areas where the resistance is reduced, and these areas coincide with the focusing trajectories), they posses some additional features which were not observed experimentally. The major difference is that the simulated resistance maps are symmetric with respect to mirror reflection along the middle line of the system (the yellow-dashed line in the first row of Fig. 7). The cyclotron orbits imaged in the experiment $\underline{13}$ were not perfectly symmetric with respect to this transformation. This asymmetry could originate from several different sources. For example, it could come from local impurity charges trapped in the sample. Due to the electric forces coming from these charges, transmitted electrons could divert from their ideal circular trajectories. Although this is a possible explanations for the asymmetry, it is unlikely in samples sandwiched between h-BN, hence here we will discuss other possible sources.

Our initial assumption was that the asymmetry could originate from a difference in widths of the two focusing leads (the $2^{\text {nd }}$ and the $3^{\text {rd }}$ lead). We tested this by changing the width of the $3^{\text {rd }}$ lead, and recalculating some of the resistance maps of Fig. 7 Using a wider $\left(l=1.2 l_{\mathrm{L}}\right)$, or a narrower $\left(l=0.8 l_{\mathrm{L}}\right) 3^{\text {rd }}$ lead did not significantly change the symmetry of the resistance maps and can not account for what is observed in the experiment. Our second assumption was that the asymmetry originates from an asymmetry in the tip-induced potential. An uneven distribution of charges on top of the tip, or a tip not properly aligned to the vertical $(z)$ axis would create an anisotropic image-charge density, and consequently an anisotropic tip potential. We tested this by modifying the eccentricity of an elliptic charge density, but the obtained resistance maps were not significantly modified. In general, we could not reproduce the measured resistance asymmetries in an impurity-free four-terminal device.

Since the original experiment was performed in a sixterminal device, in order to check how resistance maps change for different configurations of the voltage measuring probes, we additionally add two new leads to our system. We label these new leads as lead 5 , and lead 6 , and they are placed opposite to the two focusing leads. Our system is now a symmetric, six-terminal Hall bar often used in standard quantum Hall measurements.

Resistance maps of this system are shown in Fig. 9, for three different cases. The first row presents data for 


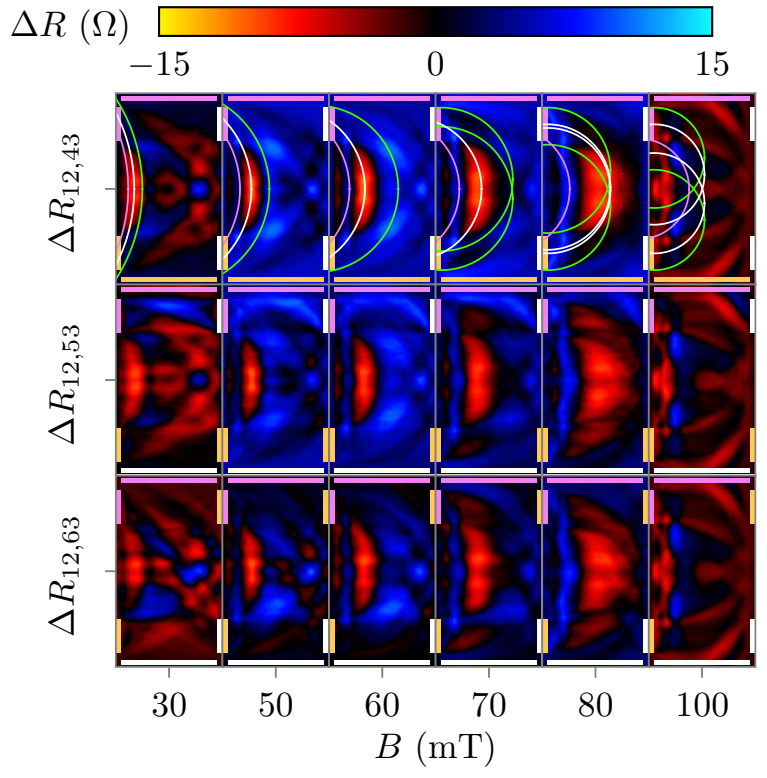

FIG. 9. (Color online) SGM resistance maps of a six-terminal device for different measurement configurations: $\Delta R_{12,43}$ (first row), $\Delta R_{12,53}$ (second row), $\Delta R_{12,63}$ (third row), and for different magnetic fields (columns). Leads colored in violet are source and drain leads for the current, and leads colored in orange are voltage probes (leads colored in white were not used in the calculations). Global electron density is $n=8.1 \times 10^{11} \mathrm{~cm}^{-2}$, and maximal tip-induced change in the charge density is $\Delta n=5 \times 10^{11} \mathrm{~cm}^{-2}$. All resistances are obtained using the quantum (tight-binding) approach. Resistance $R_{0}$ is calculated and subtracted from every inset, similarly as in Fig. 7 .

the same probe configuration as in Fig. [7 but now with two new leads included. When compared with Fig. 7 the new leads do not significantly modify the calculated resistances. The region of suppressed resistance is still delimited with two cyclotron orbits connecting the outer and inner corners of the two focusing leads (violet and green curves in the first row of Fig. 91), and maximal resistance suppression is still determined by the orbits connecting the middle lines of the two focusing leads (the white curves). The second row in Fig. 9 corresponds to probe configuration actually used in the experiment. $\underline{\underline{13}} \mathrm{~A}$ slight asymmetry is introduced by keeping the current probes (leads 1 and 2), and changing the voltage probes (from measuring $V_{43}$, to measuring $V_{53}$ ). Although the suppressed region is still fairly symmetric, an asymmetry is evident if we compare upper and lower parts of the $R$-maps. The third row shows $R$-maps obtained by measuring the voltage across the device $\left(V_{63}\right)$. A clear asymmetry is evident for lower fields. In general, the measured voltage depends mostly on how much of the electron current is scattered into the voltage leads. For stronger fields, most of the current is located away from the new leads, on the lower edge, and therefore the scattering is negligible.

Since we demonstrated that most of the transport in this system is determined by considering electron orbits that connect the $2^{\text {nd }}$ lead with the tip, the interface between the focusing leads and the main region might also play a significant role. Here, we considered the focusing leads connected to the main region with perfect $\left(90^{\circ}\right.$ degrees) corners, but due to an imperfect etching, these corners might be more smooth, thus allowing for some additional effects (e.g. new direct trajectories from the $2^{\text {nd }}$ lead to the tip).

\section{SUMMARY}

To summarize, we performed simulations of the scanning gate measurements in graphene magnetic focusing devices. Two methods (quantum and classical) were used to obtain the system transmissions. These transmissions were then applied in the (multi-terminal) LandauerBüttiker formula to calculate the device resistances. In order to perform the quantum simulations, the graphene tight-binding Hamiltonian needed to be properly scaled.

In case without the SGM tip, the focusing resistance $R_{m}(E, B)$ reveals three focusing peaks, which could be connected with three cyclotron radii. These radii were calculated using the distance between the two focusing leads, but only after including the finite widths of the focusing leads.

Depending on the voltage on the back-gate and the charge accumulated on the tip, we differentiate between six different regimes in which the tip can operate. Three out of these six regimes are unique. In general, due to the large system size, all the features of the resistance maps are captured with the classical model, and can be explained by tip influencing the direct cyclotron orbits coming from the $2^{\text {nd }}$ lead. Our results show that the largest change in the resistance is obtained for tip operating in the mixed regime (simultaneously repelling and focusing electrons). The spatial asymmetry in experimentally obtained $R$-maps can be partially explained by the specific configuration of the voltage probes, but we do not rule out other sources, such as charged impurities or edge imperfections produced during the etching process.

\section{ACKNOWLEDGEMENT}

This work was supported by the Methusalem program of the Flemish government.
1 J. Berezovsky, M. F. Borunda, E. J. Heller, and R. M. Westervelt, Nanotechnology 21, 274013 (2010).
2 J. Berezovsky and R. M. Westervelt, Nanotechnology 21, 274014 (2010). 
${ }^{3}$ R. Jalilian, L. A. Jauregui, G. Lopez, J. Tian, C. Roecker, M. M. Yazdanpanah, R. W. Cohn, I. Jovanovic, and Y. P. Chen, Nanotechnology 22, 295705 (2011).

4 M. R. Connolly, K. L. Chiou, C. G. Smith, D. Anderson, G. A. C. Jones, A. Lombardo, A. Fasoli, and A. C. Ferrari, Appl. Phys. Lett. 96, 113501 (2010)

5 M. R. Connolly, E. D. Herbschleb, R. K. Puddy, M. Roy, D. Anderson, G. A. C. Jones, P. Maksym, and C. G. Smith, Appl. Phys. Lett. 101, 023505 (2012)

6 S. Neubeck, L. A. Ponomarenko, A. S. Mayorov, S. V. Morozov, R. Yang, and K. S. Novoselov, Physica E LowDimensional Systems and Nanostructures 44, 1002 (2012).

7 S. Schnez, J. Güttinger, M. Huefner, C. Stampfer, K. Ensslin, and T. Ihn, Phys. Rev. B 82, 165445 (2010).

8 S. Schnez, J. Güttinger, C. Stampfer, K. Ensslin, and T. Ihn, New J. Phys. 13, 053013 (2011).

9 M. R. Connolly, K. L. Chiu, A. Lombardo, A. Fasoli, A. C. Ferrari, D. Anderson, G. A. C. Jones, and C. G. Smith, Phys. Rev. B 83, 115441 (2011).

10 A. G. F. Garcia, M. König, D. Goldhaber-Gordon, and K. Todd, Phys. Rev. B 87, 085446 (2013)

11 N. Pascher, D. Bischoff, T. Ihn, and K. Ensslin, Appl. Phys. Lett. 101, 063101 (2012)

12 A. Mreńca, K. Kolasiński, and B. Szafran, Semiconductor Science and Technology 30, 085003 (2015)

13 S. Bhandari, G.-H. Lee, A. Klales, K. Watanabe, T. Taniguchi, E. Heller, P. Kim, and R. M. Westervelt, Nano Lett. 16, 1690 (2016).
14 S. Bhandari, G.-H. Lee, P. Kim, and R. M. Westervelt, ArXiv e-prints (2016), arXiv:1608.02248 [cond-mat.mes-hall].

15 S. Bhandari, A. Lin, and R. M. Westervelt, ArXiv e-prints (2016), arXiv:1611.04865.

16 S. Morikawa, Z. Dou, S.-W. Wang, C. G. Smith, K. Watanabe, T. Taniguchi, S. Masubuchi, T. Machida, and M. R. Connolly, Appl. Phys. Lett. 107, 243102 (2015).

17 R. E. P. K. E. Aidala, T. Kramer, E. J. Heller, R. M. Westervelt, M. P. Hanson, and A. C. Gossard, Nat. Phys. 3, 464 (2007).

18 S. P. Milovanović, M. R. Masir, and F. M. Peeters, J. Appl. Phys. 115, 043719 (2014).

19 K. Kolasiński, A. Mreńca-Kolasińska, and B. Szafran, Phys. Rev. B 94, 115406 (2016).

20 B. Szafran, Phys. Rev. B 84, 075336 (2011).

21 C. W. Groth, M. Wimmer, A. R. Akhmerov, and X. Waintal, New J. Phys. 16, 063065 (2014).

22 M. D. Petrović and F. M. Peeters, Phys. Rev. B 94, 235413 (2016).

23 M. Büttiker, Phys. Rev. Lett. 57, 1761 (1986).

24 D. K. Ferry, S. M. Goodnik, and J. Bird, Transport in Nanostructures (Cambridge University Press, 2009).

25 M.-H. Liu, P. Rickhaus, P. Makk, E. Tóvári, R. Maurand, F. Tkatschenko, M. Weiss, C. Schönenberger, and K. Richter, Phys. Rev. Lett. 114, 036601 (2015)

26 S. Datta, Electronic Transport in Mesoscopic Systems (Cambridge University Press, 1995).

27 S. Bhandari, Imaging Electron Flow in Graphene, PhD dissertation, Harvard University (2015). 